\title{
DETERMINACIÓN DE POLIFENOLES TOTALES Y ACTIVIDAD ANTIOXIDANTE DE EXTRACTO DE SEMILLAS DE UVAS RESIDUOS DE LA PRODUCCIÓN DE PISCOS
}

\author{
Felipe Surco-Laos ${ }^{1 *}$, Hilda Ayquipa Paucar ${ }^{1}$, Wilfredo Quispe Gamboa ${ }^{1}$, \\ Jorge García Ceccarelli ${ }^{1}$, Manuel Valle Campos $^{1}$
}

\begin{abstract}
RESUMEN
Las uvas pisqueras son 8 variedades conocidas de Vitis vinifera de las cuales se obtiene el pisco. Durante el proceso de producción del pisco se desprenden del fruto subproductos como las semillas, hollejos y otros; las semillas últimamente están cobrando especial importancia debido a su alto contenido en polifenoles y sus propiedades antioxidantes. Existen diversos métodos para la obtención de extractos y con el fin de obtener extractos con el mayor contenido de polifenoles posibles, en este estudio se hace uso de un baño ultrasonido con un sistema de solvente etanol: agua: ácido acético $(90 / 9,5 / 0,5)$, el cual luego se lleva a sequedad en un evaporador rotatorio. Se determinó el índice de polifenoles totales encontrándose entre 402 a 84/g de semilla, índice de compuestos flavonoides de 9 a 42/g de semilla; la actividad antioxidante por el método de DPPH expresado como $\mathrm{IC}_{50}$ entre 0,184 a 0,858 mg de extracto y por el método FRAP expresado como mM equivalente de trolox de 0,298 a 1,178 mg de extracto de las distintas variedades, respectivamente. El resultado obtenido nos permite proyectar la recuperación de estos residuos como posibles sustancias nutracéuticas.
\end{abstract}

Palabras clave: Semillas, Vitis vinifera, compuestos polifenólicos, actividad antioxidante.

\section{PHENOLIC COMPOUNDS AND ANTIOXIDANT ACTIVITY OF GRAPE SEEDS RESIDUES OF PISCO PRODUCTION}

\begin{abstract}
Pisco grapes are 8 known varieties of Vitis vinifera from which pisco is obtained during the pisco production process, by-products such as seeds, skins and others are released from the fruit; the seeds are lately becoming especially important due to their high content of polyphenols and their antioxidant properties. There are several methods for obtaining extracts and in order to obtain extracts with the highest possible polyphenol content in this study, an ultrasonic bath with a solvent system ethanol: water: acetic acid (90/9.5/0.5) is used, which
\end{abstract}

\footnotetext{
${ }^{1}$ Facultad de Farmacia y Bioquímica. Universidad Nacional San Luis Gonzaga, Av. Los Maestros s/n Ica. felipesurco@gmail.com
} 
is then taken to dryness in a rotary evaporator. The Total Polyphenols index was determined to be between 402 to $84 \mu \mathrm{g}$ of seed, flavonoid compound index of 9 to $42 \mu \mathrm{g}$ of seed; the antioxidant activity by the $\mathrm{DPPH}$ method expressed as $\mathrm{IC}_{50}$ between 0.184 to $0.858 \mathrm{mg}$ of extract and by the FRAP method expressed as mM equivalent of trolox from 0.298 to 1.178 $\mathrm{mg}$ of extract of the different varieties respectively. The results obtained allow us to project the recovery of these wastes as possible nutraceutical substances.

Key words: Seeds, Vitis vinifera, polyphenolic compounds, antioxidant activity.

\section{INTRODUCCIÓN}

Se consideran alimentos funcionales aquellos que aportan nutrientes que cubran las necesidades nutricionales de las personas que los consumen, además de beneficios a la salud. Un alimento considerado funcional es la uva, a la que se le atribuye múltiples cualidades debido a la presencia de compuestos polifenólicos, en especial la actividad antioxidante ${ }^{1,2,3}$. Estos compuestos polifenólicos se clasifican de manera general como fenoles y ácidos fenólicos (polifenoles no flavonoides), y flavonoides ${ }^{4}$. Los polifenoles son metabolitos secundarios de las plantas que se han asociados con diversas propiedades, taxonómicas, sensoriales, nutricionales y farmacológicas ${ }^{5}$. En muchos frutos se ha encontrado un mayor poder antioxidante en las cáscaras y/o semillas que en la porción comestible. En el proceso de la producción del pisco queda como residuo un bagazo, compuesto principalmente por semillas y hollejos que son fuentes importantes de estos compuestos. Es así como la abundante cantidad de estos residuos no tienen mayor provecho, a pesar de la importancia económica que podrían tener por su contenido de compuestos polifenólicos y posible capacidad antioxidante, como lo demuestran estudios en otras variedades como: Cabernet sauvignon ${ }^{6-8}$, Carménere ${ }^{5}$, Malvasía y Tempranillo9. A pesar de lo antes dicho no hay mayores estudios a nivel nacional que se oriente a una utilización integral de estos subproductos.

Tradicionalmente, la extracción de compuestos fenólicos se ha realizado por diferentes técnicas que involucran diversos solventes orgánicos de naturaleza polar como: agua, metanol, etanol, acetato de etilo, o mezclas de ellos ${ }^{5}$. La aplicación de ondas de ultrasonido de alta intensidad es considerada como una técnica de alto rendimiento en la extracción, al facilitar el pase de metabolitos desde el interior de las células al solvente de extracción ${ }^{10}$. El pisco, bebida alcohólica destilada de origen peruano, se produce a partir de las variedades "Torontel, Italia, Mollar, Quebranta, Negra criolla, Albilla, Moscatel y Uvina"11. En el afán de realizar un aprovechamiento integral de los subproductos del proceso, el presente estudio tiene como objetivo la determinación de polifenoles totales y actividad antioxidante del extracto de semillas de las distintas variedades de uvas pisqueras, tratando de maximizar dicha extracción mediante el uso de un baño de ultrasonido. 


\section{PARTE EXPERIMENTAL}

\section{Obtención de semillas}

Las semillas de uvas de las variedades: Italia, Torontel, Moscatel, Quebranta y Mollar se obtuvieron del bagazo de uvas cultivadas en el CITE agroindustrial de Ica empleadas para la obtención de piscos; la variedad de uva Albilla fue obtenida de una bodega del distrito de Guadalupe-Ica, la variedad Uvina del fundo La Cantera del distrito Nuevo Imperial-Cañete y la variedad Negra criolla de agroindustrias Yugo SAC del departamento de Tacna, todas estas muestras se obtuvieron entre los meses de febrero a abril del 2019.

\section{Tratamiento de semillas}

Los bagazos fueron secados por 7 días al sol y posteriormente 7 días bajo sombra para luego proceder a extraer las semillas manualmente, separándolas de pieles y escobajo. Una porción de cada una de las variedades fue procesada en un molino analítico (Ica A11) y tamizada (tamiz de 20 mesh). Las muestras (polvo de las semillas) fueron almacenadas en frascos de color ámbar a $-5{ }^{\circ} \mathrm{C}$ hasta el momento de los análisis respectivos.

\section{Caracterización de semillas}

La caracterización de las semillas de las variedades de uvas pisqueras se basó en un análisis químico proximal y determinación de micronutrientes minerales por métodos oficiales según $\mathrm{AOAC}^{12}$.

\section{Obtención de extracto}

Para la obtención de los extractos se realiza un desengrasado previo de polvo de semilla de cada variedad, el cual consistió en tomar 10,00 +/- 0,02 g, se somete a una extracción sólido-líquido con éter de petróleo por espacio de 8-10 horas según Jayaprakasha $2003^{13}$, Una vez desengrasada la muestra se realiza una extracción sólido-líquido en un equipo de baño ultrasonido ${ }^{10}$, utilizando como solvente de extracción la mezcla etanol: agua: ácido acético $(90,00 / 9,50 / 0,50)$. Se deposita en un vaso de precipitado $10 \mathrm{~g}$ de muestra y $90 \mathrm{~mL}$ del solvente de extracción, se coloca el baño de ultrasonido a alta frecuencia por espacio de 30 minutos, a una temperatura entre $30-35^{\circ} \mathrm{C}$, luego se filtra el sobrenadante, con el residuo se procede a repetir el proceso por 2 veces más; se reúnen los extractos y se concentra en un evaporador rotatorio (Büchi R-210) a $40{ }^{\circ} \mathrm{C}$ para eliminar el exceso de solvente, concentrándose hasta 50 $\mathrm{mL}$. El extracto obtenido fue almacenado en frasco de color ámbar en refrigeración $\left(5-8^{\circ} \mathrm{C}\right)$ hasta su análisis ${ }^{6}$.

\section{Determinación de compuestos fenólicos}

Los compuestos fenólicos se cuantificaron de acuerdo con la reacción que presentan con el reactivo de Folin-Ciocalteu (tungstofosfato y molibdofosfato), el cual se reduce en solución alcalina, formando un producto de color azul que puede ser detectado a $760 \mathrm{~nm}^{7}$. Los datos fueron interpolados con la curva de calibración realizada con el ácido gálico (Sigma-Aldrich) como estándar. Los resultados se expresan en mg de ácido gálico/g semilla. 


\section{Índice de polifenoles totales (IPT)}

La obtención del índice de polifenoles totales se realizó por la medida de la absorbancia a $280 \mathrm{~nm}$ (UV) del extracto acuoso diluido, de acuerdo a la absorbancia característica del núcleo bencénico propio de los compuestos polifenólicos. El extracto obtenido se diluye 1:100 con agua ultrapura (millipore) y se realiza la medida de absorbancia a $280 \mathrm{~nm}$ en un espectrofotómetro Uv-vis con una celda de cuarzo. El índice se calcula como:

IPT $=\mathrm{Abs}_{(280)} \times 100$ y se realiza el cálculo respectivo para reportar el índice por gramo de semilla .

\section{Índice de contenido de flavonoles}

El contenido de flavonoles se estima por la medida de la absorbancia a $365 \mathrm{~nm}$. El extracto se diluye 1:100 con agua ultrapura (millipore) y se realiza la medida de absorbancia. El índice de flavonoles se calcula como:

$\mathrm{ICF}=\mathrm{Abs}_{(362)} \mathrm{x} 100$ y se realiza el cálculo respectivo para reportar el índice por gramo de semilla ${ }^{8}$.

\section{Determinación de actividad antioxidante por el método DPPH}

La capacidad de captación del radical libre DPPH fue determinada siguiendo el método descrito por Brand Williams, con pequeñas modificaciones. Se prepara una solución del radical libre DPPH a una concentración $100 \mathrm{mM}$ en metanol y se determina su absorbancia a $517 \mathrm{~nm}$ (la absorbancia debe estar entre 0,9 y 1,1). $0,1 \mathrm{~mL}$ de solución de los extractos de diferentes concentraciones fueron adicionada a $2,9 \mathrm{~mL}$ de la solución metanólica de DPPH, se agita en un vortex y se deja reposar en la oscuridad por 30 minutos. Una muestra control con el mismo volumen del solvente fue usado como blanco. Después de la reacción, la absorbancia fue medida a $517 \mathrm{~nm}$ para determinar la absorbancia remanente de la solución de DPPH. El porcentaje de inhibición de la absorbancia del radical DPPH es calculada como: $\%$ Inh $=\left(\left(\mathrm{Abs}_{\text {blanco }}-\mathrm{Abs}_{\text {muestra }}\right) / \mathrm{Abs}_{\text {blanco }}\right) \times 100$

El valor de IC50 es calculado usando una ecuación de regresión del porcentaje de inhibición para comparación ${ }^{14}$.

\section{Determinación de la actividad antioxidante por método FRAP}

Se siguió el procedimiento descrito por Benzie y Strain, con ligeras modificaciones. Para iniciar el análisis se prepara el reactivo de trabajo, que consiste en una mezcla de tampón acetato de sodio $300 \mathrm{mM}$ (pH =3,6), 2, 4, 6-Tri (2-pyridyl)-s-triazine (TPTZ) $10 \mathrm{mM}$ en $\mathrm{HCl}$ $40 \mathrm{mM}$ y tricloruro férrico (FeCl3. 6H2O) $20 \mathrm{mM}$ en una proporción 10:1:1 (v:v:v). Una vez preparado, se añade $3 \mathrm{~mL}$ de este reactivo en una cubeta, y se mide la absorbancia a $593 \mathrm{~nm}$. Posteriormente, se agrega $100 \mu \mathrm{L}$ de cada una de las soluciones de los extractos de diferentes concentraciones y se agita en un vórtex durante 30 segundos. Después de 6 minutos de incubación a temperatura ambiente se realiza la lectura de absorbancia nuevamente a $593 \mathrm{~nm}$, a la que se resta el valor de la absorbancia inicial. Las muestras se ensayarán por triplicado. De manera similar se trabaja con soluciones de Trolox en un rango de 0,0625 a $1 \mathrm{mM}$ como patrón de comparación, con los valores se realiza una curva de regresión frente a la cual se extrapolan los valores de las muestras. La actividad se expresa como $\mathrm{mM}$ equivalentes de trolox $(\mathrm{TEAC})^{15}$. 


\section{RESULTADOS Y DISCUSIÓN}

Tabla 1. Determinación del análisis químico proximal de las semillas de uvas pisqueras $(\mathrm{g} / 100 \mathrm{~g})$.

\begin{tabular}{lcrcrc}
\hline $\begin{array}{l}\text { Variedad de } \\
\text { uva }\end{array}$ & Humedad & Cenizas & Proteína & Grasa & Carbohidratos \\
\hline Mollar & $7,18 \pm$ & $2,20 \pm$ & $11,24 \pm$ & $9,73 \pm$ & \multirow{2}{*}{09,65} \\
& $0,14^{\mathrm{c}}$ & $0,02^{\mathrm{c}}$ & $0,87^{\mathrm{a}}$ & $0,62^{\mathrm{b}}$ & \\
Negra criolla & $7,64 \pm$ & $2,00 \pm$ & $10,08 \pm$ & $5,16 \pm$ & 75,12 \\
Quebranta & $0,23^{\mathrm{c}}$ & $0,03^{\mathrm{c}}$ & $0,63^{\mathrm{a}}$ & $0,72^{\mathrm{d}}$ & \\
& $11,47 \pm$ & $1,79 \pm$ & $8,48 \pm$ & $6,29 \pm$ & 71,97 \\
Uvina & $0,04^{\mathrm{a}}$ & $0,02^{\mathrm{d}}$ & $0,28^{\mathrm{b}}$ & $0,54^{\mathrm{c}}$ & \\
& $8,30 \pm$ & $2,77^{\mathrm{c}} \pm$ & $9,99 \pm$ & $8,56 \pm$ & 70,38 \\
& $0,26^{\mathrm{b}}$ & $0,04^{\mathrm{a}}$ & $0,45^{\mathrm{a}}$ & $0,43^{\mathrm{b}}$ & \\
Moscatel & $7,41 \pm$ & $2,57 \pm$ & $8,92 \pm$ & $11,20 \pm$ & 69,90 \\
& $0,21^{\mathrm{c}}$ & $0,04^{\mathrm{b}}$ & $0,17^{\mathrm{b}}$ & $0,28^{\mathrm{a}}$ & \\
Torontel & $7,55 \pm$ & $2,40 \pm$ & $9,40 \pm$ & $7,18 \pm$ & 73,47 \\
& $0,13^{\mathrm{c}}$ & $0,03^{\mathrm{b}}$ & $0,23^{\mathrm{b}}$ & $0,32^{\mathrm{c}}$ & \\
Italia & $7,38 \pm$ & $2,47 \pm$ & $7,79 \pm$ & $7,14 \pm$ & 75,22 \\
& $0,09^{\mathrm{c}}$ & $0,02^{\mathrm{b}}$ & $0,28^{\mathrm{c}}$ & $0,63^{\mathrm{c}}$ & \\
Albilla & $8,37 \pm$ & $2,17 \pm$ & $9,22 \pm$ & $9,26 \pm$ & 70,98 \\
& $0,16^{\mathrm{b}}$ & $0,03^{\mathrm{c}}$ & $0,25^{\mathrm{b}}$ & $0,37^{\mathrm{b}}$ & \\
\hline
\end{tabular}

Letras iguales significa que no hay diferencia estadística.

El valor de carbohidratos por cálculo

Tabla 2. Contenido de micronutrientes minerales en semillas de uvas pisqueras.

\begin{tabular}{|c|c|c|c|c|c|c|}
\hline \multirow{2}{*}{$\begin{array}{l}\text { Variedad } \\
\text { de uva }\end{array}$} & \multicolumn{6}{|c|}{ Micronutrientes } \\
\hline & $\begin{array}{c}\mathrm{Ca} \\
\mathrm{mg} / \mathrm{kg}\end{array}$ & $\begin{array}{c}\mathrm{Mg} \\
\mathrm{mg} / \mathrm{kg}\end{array}$ & $\begin{array}{c}\mathrm{Fe} \\
\mathrm{mg} / \mathrm{kg}\end{array}$ & $\begin{array}{c}\mathrm{Zn} \\
\mathrm{mg} / \mathrm{kg}\end{array}$ & $\underset{\mathrm{mg} / 100 \mathrm{~g}}{\mathrm{~K}}$ & $\begin{array}{c}\mathrm{P} \\
\mathrm{mg} / 100 \mathrm{~g}\end{array}$ \\
\hline Mollar & $\begin{array}{c}2283 \pm \\
76,1^{\mathrm{b}}\end{array}$ & $\begin{array}{c}871 \pm \\
9,6^{\mathrm{d}}\end{array}$ & $\begin{array}{c}69,2 \pm \\
4,3^{c}\end{array}$ & $\begin{array}{c}14,0 \pm \\
3,0 \mathrm{~b}\end{array}$ & $\begin{array}{c}187 \pm \\
6,1^{\mathrm{d}}\end{array}$ & $\begin{array}{c}934 \pm \\
5,6^{\mathrm{b}}\end{array}$ \\
\hline Negra criolla & $\begin{array}{c}1206 \pm \\
34,2^{\mathrm{e}}\end{array}$ & $\begin{array}{c}1102 \pm \\
31,4^{\mathrm{c}}\end{array}$ & $\begin{array}{c}67,3 \pm \\
4,9^{c}\end{array}$ & $\begin{array}{c}12,9 \pm \\
1,7^{\mathrm{c}}\end{array}$ & $\begin{array}{l}468 \pm \\
22,4^{\mathrm{a}}\end{array}$ & $\begin{array}{l}667 \pm \\
11,3^{\mathrm{c}}\end{array}$ \\
\hline Quebranta & $\begin{array}{c}2497 \pm \\
73,5^{\mathrm{b}}\end{array}$ & $\begin{array}{l}887 \pm \\
21,0^{d}\end{array}$ & $\begin{array}{c}84,2 \pm \\
2,4^{b}\end{array}$ & $\begin{array}{c}10,1 \pm \\
0,9^{\mathrm{c}}\end{array}$ & $\begin{array}{c}199 \pm \\
7,8^{d}\end{array}$ & $\begin{array}{l}697 \pm \\
23,2^{\mathrm{c}}\end{array}$ \\
\hline Uvina & $\begin{array}{c}1848 \pm \\
45,4^{\mathrm{c}}\end{array}$ & $\begin{array}{c}1227 \pm \\
31,2^{\mathrm{c}}\end{array}$ & $\begin{array}{c}104 \pm \\
5,9^{\mathrm{a}}\end{array}$ & $\begin{array}{c}17,9 \pm \\
2,6^{\mathrm{a}}\end{array}$ & $\begin{array}{l}436 \pm \\
11,3^{\mathrm{b}}\end{array}$ & $\begin{array}{c}1199 \pm \\
24,3^{\mathrm{a}}\end{array}$ \\
\hline Moscatel & $\begin{array}{c}1500 \pm \\
61,1^{\mathrm{d}}\end{array}$ & $\begin{array}{c}2405 \pm \\
16,9^{\mathrm{a}}\end{array}$ & $\begin{array}{c}62,0 \pm \\
2,8^{\mathrm{d}}\end{array}$ & $\begin{array}{c}12,1^{ \pm} \\
1,1^{\mathrm{c}}\end{array}$ & $\begin{array}{c}241 \pm \\
5,7^{\mathrm{c}}\end{array}$ & $\begin{array}{l}519 \pm \\
35,6^{d}\end{array}$ \\
\hline Torontel & $\begin{array}{c}1342 \pm \\
24,3^{\mathrm{e}}\end{array}$ & $\begin{array}{c}1066 \pm \\
31,4^{d}\end{array}$ & $\begin{array}{c}63,2 \pm \\
4,5^{\mathrm{d}}\end{array}$ & $\begin{array}{c}11,2 \pm \\
0,7^{\mathrm{c}}\end{array}$ & $\begin{array}{l}284^{ \pm} \\
12,4^{\mathrm{c}}\end{array}$ & $\begin{array}{l}624 \pm \\
31,1^{c}\end{array}$ \\
\hline Italia & $\begin{array}{c}1123 \pm \\
35,7^{\mathrm{d}}\end{array}$ & $\begin{array}{c}1303^{ \pm} \\
17,5^{\mathrm{c}}\end{array}$ & $\begin{array}{c}68,5 \pm \\
2,1^{\mathrm{c}}\end{array}$ & $\begin{array}{c}12,4 \pm \\
0,9^{\mathrm{c}}\end{array}$ & $\begin{array}{c}414 \pm \\
8,1^{b}\end{array}$ & $\begin{array}{l}703 \pm \\
23,8^{b}\end{array}$ \\
\hline Albilla & $\begin{array}{c}3054 \pm \\
31,4^{\mathrm{a}}\end{array}$ & $\begin{array}{c}2005^{ \pm} \\
32,6^{\mathrm{b}}\end{array}$ & $\begin{array}{c}72,5 \pm \\
2,9^{\mathrm{c}}\end{array}$ & $\begin{array}{c}17,5 \pm \\
2,4^{\mathrm{a}}\end{array}$ & $\begin{array}{l}492 \pm \\
14,2^{a}\end{array}$ & $\begin{array}{l}570 \pm \\
25,6^{d}\end{array}$ \\
\hline
\end{tabular}

Letras iguales significa que no hay diferencia estadística. 
Tabla 3. Rendimiento de extracto de las semillas de uvas pisqueras.

\begin{tabular}{cccc}
\hline $\begin{array}{c}\text { Variedad de } \\
\text { uva }\end{array}$ & $\begin{array}{c}\text { Rendimiento } \\
\text { Peso de } \\
\text { semilla }(\mathrm{g})\end{array}$ & $\begin{array}{c}\text { Peso seco de } \\
\text { extracto }(\mathrm{g})\end{array}$ & $\begin{array}{c}\text { Porcentaje en } \\
\text { g/100g }\end{array}$ \\
\hline Quebranta & 10,00 & 0,3341 & $3,34 \pm 0,07^{\mathrm{a}}$ \\
Mollar & 10,01 & 0,2523 & $2,52 \pm 0,04^{\mathrm{b}}$ \\
Uvina & 10,03 & 0,261 & $2,60 \pm 0,07^{\mathrm{b}}$ \\
Negra criolla & 10,01 & 0,1884 & $1,88 \pm 0,12^{\mathrm{c}}$ \\
Moscatel & 10,04 & 0,3955 & $3,95 \pm 0,07^{\mathrm{b}}$ \\
Torontel & 10,07 & 0,1855 & $1,86 \pm 0,04^{\mathrm{d}}$ \\
Italia & 10,01 & 0,248 & $2,48 \pm 0,07^{\mathrm{c}}$ \\
Albilla & 10,03 & 0,544 & $5,44 \pm 0,12^{\mathrm{a}}$ \\
\hline
\end{tabular}

Letras iguales significa que no hay diferencia estadística.

Tabla 4. Determinación de índice de polifenoles totales, polifenoles totales e índice de compuestos flavonoides en extracto de semillas de uvas pisqueras

\begin{tabular}{cccc}
\hline $\begin{array}{c}\text { Variedad de } \\
\text { uva }\end{array}$ & IPT/g semilla & $\begin{array}{c}\text { Compuestos polifenólicos } \\
\text { Polifenoles totales } \\
\text { mgEAG/g }\end{array}$ & ICF/g semilla \\
\hline Quebranta & $210,5 \pm 6,7^{\mathrm{b}}$ & $1,96 \pm 0,07^{\mathrm{c}}$ & 24,5 \\
Mollar & $171,8 \pm 4,2^{\mathrm{c}}$ & $2,31 \pm 0,05^{\mathrm{b}}$ & 27,5 \\
Uvina & $226 \pm 6,8^{\mathrm{b}}$ & $1,61 \pm 0,08^{\mathrm{d}}$ & 33 \\
Negra criolla & $44,5 \pm 0,9^{\mathrm{e}}$ & $0,32 \pm 0,03^{\mathrm{e}}$ & 9 \\
Moscatel & $402,7 \pm 6,7^{\mathrm{a}}$ & $2,53 \pm 0,09^{\mathrm{a}}$ & 42,3 \\
Torontel & $84 \pm 4,2^{\mathrm{d}}$ & $1,26 \pm 0,05^{\mathrm{d}}$ & 13,5 \\
Italia & $222 \pm 6,8^{\mathrm{b}}$ & $2,08 \pm 0,08^{\mathrm{b}}$ & 31 \\
Albilla & $52,5 \pm 0,9^{\mathrm{e}}$ & $1,94 \pm 0,03^{\mathrm{c}}$ & 9,5
\end{tabular}

Letras iguales significa que no hay diferencia estadística. 
Tabla 5. Determinación de la actividad antioxidante en los extractos de semillas de uvas pisqueras

\begin{tabular}{ccc}
\hline $\begin{array}{c}\text { Variedad de } \\
\text { uva }\end{array}$ & $\begin{array}{c}\text { Actividad antioxidante } \\
\text { DPPH }\end{array}$ & $\begin{array}{c}\text { FRAP } \\
\text { mg extracto/mMTE }\end{array}$ \\
\hline Quebranta & $0,375 \pm 0,023^{\mathrm{b}} / \mathrm{mg} \mathrm{de} \mathrm{extracto}^{\mathrm{b}}$ & $0,707 \pm 0,019^{\mathrm{b}}$ \\
Mollar & $0,345 \pm 0,027^{\mathrm{b}}$ & $0,675 \pm 0,021^{\mathrm{b}}$ \\
Uvina & $0,184 \pm 0,014^{\mathrm{d}}$ & $0,220 \pm 0,012^{\mathrm{e}}$ \\
Negra criolla & $0,858 \pm 0,027^{\mathrm{a}}$ & $1,178 \pm 0,034^{\mathrm{a}}$ \\
Moscatel & $0,208 \pm 0,013^{\mathrm{d}}$ & $0,427 \pm 0,013^{\mathrm{c}}$ \\
Torontel & $0,194 \pm 0,017^{\mathrm{d}}$ & $0,298 \pm 0,021^{\mathrm{d}}$ \\
Italia & $0,282 \pm 0,024^{\mathrm{c}}$ & $0,399 \pm 0,012^{\mathrm{c}}$ \\
Albilla & $0,260 \pm 0,049^{\mathrm{c}}$ & $0,423 \pm 0,023^{\mathrm{c}}$
\end{tabular}

Letras iguales significa que no hay diferencia estadística.

En el presente estudio se investigó el contenido de polifenoles totales y actividad antioxidante en el extracto de semillas de las ocho variedades de uvas utilizadas para la producción de pisco, producto de bandera peruano. Las semillas de las variedades Torontel, Moscatel, Albilla, Italia, Quebranta, Mollar y Uvina fueron obtenidas inmediatamente después de la expresión para obtener el mosto respectivo; mientras que la variedad Negra criolla se obtuvo después de días del proceso de fermentación del mosto (como es costumbre la fermentación en la región de Tacna). Todos los bagazos fueron secados de manera similar al sol y luego bajo sombra pretendiendo neutralizar los posibles procesos enzimáticos remanentes. En las tablas 1 y 2 se observa los valores de las determinaciones químico proximal y minerales realizadas a las semillas de cada variedad de uvas pisqueras, siendo mayores a los valores de la variedad Cabernet Sauvignon reportado por Mojica y Pérez et al $2019^{8}$ (en cenizas y nitrógeno llevados a base seca), estas determinaciones se realizaron con el objetivo de caracterizarlas; sin embargo, estos valores se deben tomar con precaución, ya que son resultados de la producción de un mismo año y zona. Teniendo conocimiento que las extracciones por ultrasonido mejoran los rendimientos de los metabolitos secundarios ${ }^{10}$, para obtener una mayor riqueza polifenólica y actividad antioxidante de los extractos se fijaron los valores experimentales como temperatura $30-35^{\circ} \mathrm{C}$, la intensidad de ultrasonido (alta intensidad del equipo) y el tiempo de extracción fue de 30 minutos en cada extracción (se realizó tres extracciones por muestras). En la tabla 3, se aprecia que los valores del rendimientos de los extractos varían en cada una de las variedades, va de 1,88 a 5,43 \%, lo que podemos atribuir a la naturaleza misma de las semillas, puesto que todas fueron sometidas a similar procedimiento, Berradre et al $2013^{9}$ encontraron un rendimiento entre 5 y $6 \%$ en las variedades Tempranillo y Malvasía por el método de Soxhlet con una variación entre 7 a 9 horas de extracción, con 
una diferencia en el solvente pues usaron metanol a diferencia del presente trabajo donde usamos el etanol, pero Jayaprakasha et al $2003^{13}$ también señalan un rendimiento entre 5 y 6 $\%$ empleando diferentes tipos de solventes. Respecto al índice de polifenoles totales e índice de compuestos flavonoides la variedad Moscatel resultó con mayores valores, seguida de la Uvina, con valores dentro de los reportados en otros estudios ${ }^{9,13}$; Mojica y Pérez reportan para la variedad Cabernet valores de IPT menor (117) y ICF valor promedio (33) que la mayoría de los casos de este estudio; en la determinación de polifenoles totales se encontró una coincidencia en la variedad de mayor contenido (Moscatel) y de menor contenido (Negra criolla) con respecto a las determinaciones de índice de fenoles y flavonoides, en lo referente al contenido de polifenoles totales los valores encontrados son menores a lo reportado por Mojica y Pérez 2019 (12,3 mg/g pero determinado en orujo $)^{8}$. La actividad antioxidante, como se muestra en la tabla 5, todos los extractos muestran que tienen capacidad de inhibitoria del radical DPPH, siendo los más activos Uvina y Moscatel al presentar menores valores de $\mathrm{IC}_{50}$, lo que se puede correlacionar con presencia de compuestos bioactivos según la tabla anterior, y la variabilidad de los resultados podría deberse a los diferentes tipos de compuestos polifenólicos que presenten cada variedad de uva, o que estos compuestos se encuentren enlazados a otros tipos de sustancias que lo hagan más insolubles o complejos, para permitirles una reacción directa con el radical DPPH. Considerando el mecanismo de reacción por lo cual actúa este radical nos permite suponer la presencia de sustancias que actúan como dadores de átomos de hidrógenos, así como captadores de electrones libres. La actividad antioxidante por el método FRAP es uno de los más usados para probar el poder reductor de los compuestos antioxidantes; sin embargo, los valores obtenidos no pueden ser extrapolados a condiciones fisiológicas, en la tabla 5 se muestran los resultados obtenidos por este método en los extractos de semillas, siendo más efectivas las variedades Uvina y Torontel al requerir menos miligramos del extracto para alcanzar una equivalencia de un milimol de trolox, lo que nos indica que poseen compuestos donadores de electrones, que es el mecanismo en que se basa este método. El mayor valor de equivalente de trolox obtenido en la variedad Negra criolla podría explicarse por el hecho que al estar sometidas al proceso de fermentación en la presencia de ácidos y diversas enzimas liberan mayor cantidad de estos compuestos hacia el mosto quedando ellas deficientes ${ }^{16}$.

\section{CONCLUSIONES}

Los resultados nos permiten concluir que la semilla de uvas de mayor rendimiento, desde el punto de vista del análisis químico proximal y mineral, es la variedad Albilla, y que los extractos de semillas poseen una alta capacidad antioxidante, sobresaliendo la variedad Torontel y Uvina, lo cual se relaciona con el contenido de compuestos fenólicos y flavonoides, lo que nos lleva a considerarlas como alimentos funcionales. 


\section{REFERENCIAS BIBLIOGRÁFICAS}

1. Trescastro-López E, Bernabeu-Mestre J. Alimentos funcionales: ¿necesidad o lujo? Rev Esp Nutr Hum Diet. 2015; 19(1): 1 - 3.

2. Cano M, Sánchez-Moreno C, Pascual-Teresa S, Ancos B. Nuevas tecnologías de conservación de productos vegetales frescos cortados. Guadalajara, México: Logiprint Digital; 2005 pp. 289-312.

3. Molina-Quijada D, Medina-Juárez L, Gonzales-Aguilar L, Robles-Sánchez R y GámezMeza N. Compuestos fenólicos y actividad antioxidante de cáscara de uva (Vitis vinifira L) de mesa cultivada en el noreste de México. CyTA - J Food. $2010: 8$ (1): 57-63.

4. Gallego J. Servicios de vinos. Madrid:Ediciones Paraninto S.A.; 2011.

5. Cerda A. Caracterización polifenólica y de la capacidad antioxidante de orujos de cuatro variedades de Vitis vinifera L. [Tesis de pregrado]. Santiago de Chile: Universidad de Chile; 2014.

6. Paladino S, Zuritz C. Extracto de semillas de vid (Vitis vinifera L.) con actividad antioxidante: eficiencia de diferentes solventes en el proceso de extracción. Rev FCA UNCUYO. 2011: 43: 187-199.

7. Ainsworth EG. Estimation of total phenolic content and other oxidation substrates in plant tissues using folin-Ciocalteu reagent. Nat Protocol. 2007: 2: 875-877.

8. Mojica J, Pérez W. Aprovechamiento de residuos agroindustriales de la industria vinícola del valle de Sáchica. [Internet]. Servicio Nacional de Aprendizaje. SENA2019. [accesado 12 ene 2019]. Disponible en. https://repositorio.sena.edu.co/bitstream/11404/5389/1/ aprovechamiento_residuos_agro_vinicola_sachica.pdf

9. Berradre M, Gonzales C, Sulbarán B, Fernández V. Contenido de polifenoles y actividad antioxidante de extractos de semilla de uva (Vitis vinifera) variedad Malvasía y Tempranillo. Rev Fac Agron (LUZ). 2013; 30: 613-631.

10. Acosta J, Salomón S, Sevilla I, Nuevas L. Empleo del ultrasonido para la extracción de fracción apolar en hojas de Mangifera indica L. (árbol del mango). Rev Cubana Plant Med. 2016: 21 (3): 261-271.

11. INDECOPI. Norma Técnica Peruana 211.001-2006 -. Bebidas Alcohólicas. Pisco. Requisitos.

12. AOAC. Official Methods of Analysis. 18th Edition. Gaithersburgs, MD:Association of Official Analytical Chemists; 2006.

13. Jayaprakasha G, Selvi T, Sakariah K. Antibacterial and antioxidant activities of grape (Vitis vinifera) seed extracts. Food Res Int. 2002: 36 (2): 117-122.

14. Surco-Laos F, Tipiana R, Torres Y, Valle M, Panay J. Efectos de liofilización sobre composición química y capacidad antioxidante en pulpa de cuatro variedades de Mangifera indica. Rev Soc Quím Perú. 2017; 83(4): 412-419.

15. García A, De Pascual T, Santos C. Evaluation of the antioxidant properties of fruits. Food Chem. 2004: 84 (1): 13-18.

16. Li H, Wang X, Li P, Li Y, Wang H. Comparative Study of Antioxidant Activity of Grape (Vitis vinifera) Seed Powed Assessed by Different Methods. J Food Drug Anal. 2008; 6 (6): 1041-1049. 